\title{
Catheter Inner Diameter
}

National Cancer Institute

\section{Source}

National Cancer Institute. Catheter Inner Diameter. NCI Thesaurus. Code C150226.

The measurement of the diameter of the inner catheter tubing which goes over the guidewire. 